\title{
Ueber einen Fall von Gummiknoten der Cauda equina.
}

\author{
Von \\ Seigo Minami. \\ (Aus der dermatologischen Klinik der medizinischen Fakuläat au Okayama)
}

\begin{abstract}
Die Rückenmarkssyphilis kommt sehr mannigfaltig vor, jedoch ist nur ein einziger Gummiknoten an der Cauda equina ausserordentlich selten. Delafield ${ }^{1)}$ beschrieb eine kāsige Geschwulst der Cauda equina, wahrscheinlich einen Gummiknoten, der mehrere Spinalnerven umschloss. Schlesinger ${ }^{2)}$ sah einen Fall von syphilitischer Caudaerkrankung, welche aber nur klinisch diagnostiziert wurde. Herr Prof. L. Pick ${ }^{3)}$ demonstrierte einen Fall von Gummiknoten der Cauda equina in der Berliner dermatologischen Gesellschaft im Jahre 1923. Er überliess mir freundlichst dieses Material, zu untersuchen, als ich im Jahre 1922 in seinem Institut des städtischen Krankenhauses im Friedrichshain-Berlin studierte. Damals konnte ich es leider wegen meiner kurzen Zeit nicht publizieren. Für die Anregung zu dieser Arbeit bin ich ihm zu herzlichem Dank verbunden. Ich habe diesen Fall klinisch nicht beobachtet und zitiere hier nur seine Krankengeschichte.
\end{abstract}

Krankengeschichte. R. R. 6.1 jalıriger Kellner aus Berlin.

Anlamnese: Familienanamnese ohne Besonderheiten. Vor 20 Jahren litt er an Lues (Schanker ?). Seit etwa 10 Wochen klagt er über wechselnd stark anhaltende Schmerzen und gelegentliches Gefühl der Empfindungslosigkeit in den Beinen von der Hüfte bis zum Fuss. Seit etwa 10. September 1920 trat Incontinentia urinae et alvi auf. Letzter Arbeitstag: 8. September 20. Am 16. September Aufnahme im stådtischen Krankenhaus im Friedrichshain.

Status praesens: Schwlchlicher Mann in massigem Ernahrungszustand. Haut und sichtbare Schleimhaut blass. Keine Exantheme, kein Oedem, keine Drüsenschwellung, Zunge 
bräunlich belegt, måssig feucht. Rachenhöhle o. B.

Lunge: voller Klopfschall, sparliches Rasseln, besonders iiber der linken Lunge.

Herz: Grenzen normal. Erster Ton uiber der Herzpitze unrein, zweiter Ton über sâmtlichen Ostien unrein.

Puls : regelmăssig, Gefässe verhărtet.

Leib: weich, Leber, Milz nicht fühlbar.

Urin : o. B.

Nervensystem: Hirnnerven o. B. Pupillenreflex vorhanden, Linke Pupille verzogen. Normale Bauchdeckenreflex beiderseits. Patellarreflex beiderseits vorhanden, Achilles- und Sohlenreflexe beiderseits fehlend. Starke Hypalgesie im gesamten Sakralgebiet, nach abwärts zunehmend. In den unteren Lumbalsegmenten leichte Abstumpfung angegeben. D. h. vollständig aufgehobene Sensibilität (Berührung, Schmerzen und Temperatur) im Bereich von S. 4-5. Herabgesetzte, z. T. aufgehobene Berührungsemfindlichkeit im Bereich von S. 1-3, Schmerzempfindlichkeit bei lang anhaltenden Reizen erhalten. Tiefensensibilität erhalten, keine Ataxie.

Schwäche der Muskulatur der unteren Extremitäten, Stehen ohne Stütze unmöglich. Hüftbeugung und Unterschenkelstreckung beiderseits normal, Kniebeugung beiderseits schlecht, Fuss- und Zehenbeugung beiderseits unmöglich. Arrnkraft nicht geschwächt. Inkontinentia urinae et alvi.

Augenspiegeltetund : bei Durchleuchtung von vorn beiderseits, rechts der 7 , links der 6 des Zeigerblattes entsprechend, vom Rande der Pupille bis beinahe zur Mitte gehend strichförmiger, umschriebener Schatten, bei seitlicher Beleuchtung nicht sichtbar. Augenhintergrund o. B.

W. R. im Blut stark positiv. Diagnose: Myelitis sacralis.

Verlauf: Befunde unverândert, lăsst Stuhl und Urin unbemerkt ab.

23. 1X. Neosalvarsan (D I) 0.15 injiziert.

27. IX. Neosalvarsan (D I) 0.3 , Kali jodati 1.5

30. IX. Befund unverăndert. Neosalvarsan (D II) 0.6

5. X. Neosalvarsan (D II) 0.9

Im anåsthetischen Gebiet am Steissbein Haut infiltriert, gerötet, geringe Fluktuation. Gewebe in der Tiefe nekrotisch. Bei Inzision werden keinerlei Schmerzen an. gegeben. Im Urinsediment massenhafte Leukozyten. Sonstiges Befinden unverăndert.

11. X. Neosalvarsan (D III) 1.35 , Urotropin 1.5 Temperaturerhöhungen bis auf $38.5^{\circ}$, kein wesentlicher Organbefund.

15. X. Patient meint, Stuhlgang im Rektum zu fühlen und etwas zurïckhalten zu kōnnen. Urinabgang unverandert.

18. X. Neosalvarsan (D III) 1.8

20. X. Patient kaum mit Kot beschmutzt, nur mit Urin. Er kann linkes Fussgelenk etwas bewegen, desgl. grosse Zehe, sonst Nervenbefund derselbe.

23. X. Neosalvarsan (D III) 2.25 
25. X. Kann Stuhlgang halten, nicht Urin. Linker Fuss wird bewegt, desgl. Zehen, rechter Fuss unverandert. Sensibilitat: im Bereicl der 1. und 2. Sakralsegmente wird vorn auch teilweise verzögert angegeben. Unterschied von spitz und stumpf. schwer möglich. Am Sakralsegment 4. u. 5. Befund unverăndert.

Inzisionsherd zeigt keine frischen Granulationen, hat sich nur wenig verkleinert. Haut im Umkreis mazeriert.

28. X. Neosalvarsani(D IV) $\mathbf{2 . 8 5}$

4. XI. Neosalvarsan (D IV) 3.45

5. XI. Allgemeinbefinden unverandert. Rechter Fuss noch nicht beweglich. Am rechten Arm hat sich an der Injektionsstelle ein etwa wallnussgrosses Infiltrat gebildet, nicht sonderlich schmerzhaft. Feuchter Verband.

8. XI. Infiltrat kleiner geworden. Die Beine wurden elektrosiert, objektiv Befund unverăndert.

9. XI. Neosalvarsan (D IV) 4.05

12. XI. Infiltrat verschwunden. Stuhl wird gehalten, Blase immer noch ausser Funktion. Beginn einer Quecksilberschmierkur (6×5 graue Salbe).

21. XI. Auf dem linken Arm leichte Rötung und Schuppung, starkes Jucken. Arm wird deshalb aus der Kur gelassen.

22. XI. Allgemeine Rötung der Haut der Arme, in schwăcherem Masse auch der Beine. Schmierkur und Salvarsan abgesetzt.

25. XI. Jetzt die Haut des ganzen Körpers stark gerötet und geschwollen, Gesicht gedunsen, sodass Patient kaum die Lider öffnen kann. Ohren unförmig geschwollen. Urin alkalisch, Leukozyten, keine Zylinder.

28. XI. Schwellung; weiter sehr stark. Gesicht etwas weniger gedunsen. Haut mit grossen Schuppen bedeckt.

1. XII. Körpertemperatur $38^{\circ} .6$, Breidiāt. Schwellung im ganzen geringer, nur der linke Arm ist noch stark geschwollen. Auf dem Kreuzbein hat sich ein etwa handgrosser, massig tiefer Dekubitus entwickelt, der schmierigschmutzig belegt ist.

3. XII. Haut schuppt sich stark ab. Rőtung und Schwellung der Haut zurïckgegangen. Der linke Unterarm spindelförmig, bretthart geschwollen, ebenso Schwellung des linken Oberarms. Salbenverband. Starker Tremor der Hände. Körpertemperatur $39^{\circ} .2$.

5. XII. Körpertemperatur $37^{\circ} .8$. Am ganzen Körper stark schilfernde Haut. Am linken Unterund Oberarme fluktuierender Herd, durch den gelben Eiter durchschimmert (Phlegmone). Patient wird zwecks Operation zur chirurgischen Station verlegt. 4 Inzisionen im Chlorăthylrausch, es fliesst dünnfïissiger Eiter ab, in den Inzisionswunden sieht man die nekrotische Fascie. Feuchter Verband.

7. XII. Temperatur abgefallen, Wunde sezerniert māssig. Allgemeiner Zustand schlecht, Patient lăsst dauernd unter sich. Dekubitus vergrössert. Sepsis.

3. XII. Patient heute volkommen benommen. Puls 136 , klein.

9. XII. 1 Uhr vormiltags tritt unter dem Zeichen zunehmender Herzschwache der Exitus lethalis ein.

Sektionsbefund: obduziert am 11 . XII.

Aeusseres und Extremităten: $1 \mathrm{~m} .50 \mathrm{~cm}$. grosse leiche in sehr dürtigem Ernăhrungszustand, schwachem Fettpolster und schwacher Muskulatur. Haut pergamentartig verdickt mit aus. gedehnten Schuppenbildungen. Ueber dem Kreuzbein findet sich ein etwa handtellergrosser Dekubitus vom schwarzblauer Farbe, trocken, mit weit unterminierten Răndern. Am linken Ellen. bogen findet man eine kleinhandtellergrosse Phlegmone, die nach Seiten hin Oeffnungen zeigt. Totenstarre gelōst. Ausgedehnte Totenflecke an den abhăngigen Partien.

Brusthöhle: Zwerchfellstand links 4. I. C. R., rechts 5. Rippe. Tonsillen bohnengross, zerklifftet. Keblkopf stark verknőchert. Trachea, Oesophagus, Thyreoidea frei.

Herz: Dilatation beider Ventrikel. IIypertrophie links. Farbe auf dem Durchschnitt rot. 
gelh, Konsistefz derb und fest. Leichte fibröse Verdicknng des Aortensegels der Mitralis. Coronarien weit und klaffend, verdickt. Aorta von Anbeginn an mit zahlreichen narbigen Einziehungen und gelben Bucklungen, nach der Brustaorta zu geringer werdend. Durchmesser der Aorta $10.5 \mathrm{~cm}$.

Pleura beiderseits besonders in den Unterlappen mit der Brustwand flächenhaft verwachsen. Im linken Oberlappen vereinzelte frische, fetzige Auflagerungen.

Lunge: Ueber beiden Lungen verstreut zahlreiche bronchopneumonische Herde. Emphysem in den Randpartien.

Bauchhöhle: Milz 14:8:3 cm., sehr schlaff und weich. Pulpa leicht abstreichbar. Farbe auf dem Durchschnitt blaurot. Nebennieren frei, links. Rinde etwas breit.

Niere : links $11.5: 4.5: 4 \mathrm{~cm}$. Konsistenz schlaff und derb. Oberflache granuliert mit vereinzelten narbigen Einziehungen. Auf dem Durchschnitt rotgelbe, verschmalerte Rinde, verwaschene Zeichnung. Die Oberflăche zeigt vereinzelte bis stecknadelkopfgrosse $\mathrm{Cy}$ stchen. Rechte Niere $10.5: 5: 3 . \overline{\text {, }}$, Befund wie links.

Blase: Schleimhaut und Muskulatur stark verdickt. Mastdarm frei. Prostata: leicht vergrössert mit auffallend gelben Einlagerungen. Darm frei. Magen, Duodenum und Pancreas frei.

Leber : $23: 20: 6.5 \mathrm{~cm}$., Konsitenz missig derb und fest. Deutliche Zeichnung, rôtlichgelbe Farbe auf dem Durchschnitte. Papille, Gallengange frei. Gallenblase enthalt måssig dulnnflïssigen Inhalt. Ein grosser Teil des grossen Netzes ist durch den Leistenkanal in den Hodensack eingestulpt. Feste Verwachsungen.

Kopf und Rückenmark: Gehirn frei. Rulckenmark : makroskopisch von normaler Konsistenz und deutlicher Zeichnung von grauer und weisser Substanz. Starke Injektion der Gefässe durch Hypostase. Keine Injektion der Hăute.

Die Eröffnung des unteren Fndes des Duralsacks ergibt, dass in der Cauda equina eine Anzahl von Nervenbündeln in eine Schwiele eingelagert ist und in diese Schwiele sich eine halbbohnengrosse, zackig begrenzte, schwefelgelbe kāsige Masse befindet. Cauda equina z. $T$. injiziert.

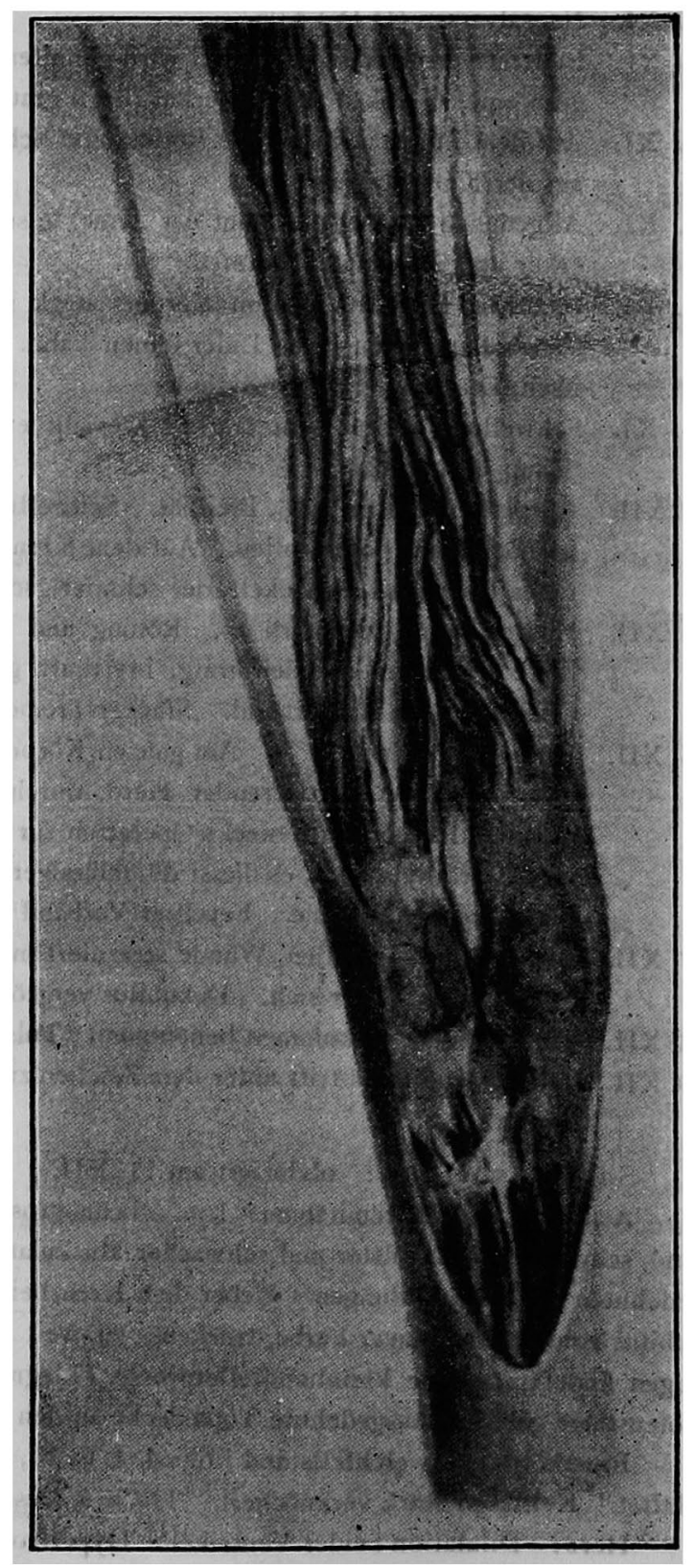




\section{Anatomische Diagnose:}

Mesaortitis luetica. Dilatation beider Herzventrikel. Hypertrophie des linken Herzens. Parenchymatöse Degeneration des Herzens und der Leber. Coronarsklerose. Arteriosklerose. Pleuritis fbrinosa, links. Bronchopneumonische Herde in beiden Lungen. Septische Milz. Arteriosklerotische Schrumpfniere. Hernia inguinalis dextra. Phlegmone am linken Arm. Decubitus. Gummiknoten der Canda equina.

Histologische Untersuchung des Gummiknotens der Cauda equina : $\mathrm{Da}$ das Material wegen seiner Seltenheit konserviert werden sollte, wurde mir nur ein kleiner Teil ausgehändigt; ich habe daher nicht alles histologisch untersuchen können. Man findet diffuse amorphe Koagulationsnekrose, in welcher noch mässige Lymphozyten zn sehen sind. Nach dem Rande zu vermehren sich diese an Zahl. In diesem Nekroseherd kann man keine Spur von Nervenfasern konstatieren, degegen verlaufen an den beiden Enden des Gummiknotens mehrere Nervenfasern in normalen Bündeln. In die Nervenbündel treten Lymphozyteninfiltrationen hinein. In der Umgebung des Gummiknotens findet man hyperämische Meningen. Leider ist die histologische Untersuchung nicht eingehend, doch ist die Diagnose des Gummiknotens nach dem klinischen Verlauf und W. R. sicher festzustellen.

Dieser einzeln aufgetretene Gummiknoten entstand wahrscheinlich auf der Meninge und erzeugte durch sein Wachstum Druckatrophie der Nervenbündel. Klinische Erscheinungen sollen je nach der Höhe und der Grösse des Gummiknotens wechseln. Im allgemeinen tritt zuerst Reizsymptom, wie ausstrahlende, quālende Schmerzen auf, darauf Lähmungssymptome im Sakralgebiet, wie Störungen der Sensibilität, der motorischen Kraft, der Blasen-, Mastdarm- und Geschlechtsfunktionen u. a. Kurz, die Erscheinungen sind ganz āhnlich mit Tumor oder anderen Lāsionen in diesem Gebiet, aber Anamnese, andere fi ühere oder noch vorhandene syphilitische Symptome, schliesslich serologische Reaktion können die Diagnose feststellen. 


\section{Literatur.}

1) Delafield, American Journ. of dermatology and syphilis. 1873. zit. von Herxheimer im Ergebnisse d. allg. Pathologie und pathologischen Anatomie des Menschen und der Tiere. 11, 1906.

2) Schlesinger, H., Das Zerrungssymptom bei Erkrankungen der Cauda equina. Neurologisches Centralblatt. 34, 1915.

3) Pick, L., Dermatolog. Zeitschr. II. 5-6, Bd. 37, 1923. 